\title{
INVESTIGATION ON MECHANICAL VIBRATION OF DOUBLE-WALLED CARBON NANOTUBES ON WINKLER FOUNDATION WITH LENGTH EFFECTS VIA DTM
}

\author{
B. Ravi Kumar* and K. Palaksha Reddy \\ School of Mechanical Engineering, SASTRA University, Thanjavur-613401,Tamilnadu (India) \\ *E-mail : ravikumar@mech.sastra.edu
}

\begin{abstract}
In the present study, vibration response of double walled carbon nanotubes embedded in a Winkler foundation is investigated using differential transform method (DTM). Simply supported, clamped - Hinged and clampedclamped boundary conditions are employed to analyze the natural frequencies. The effect of length of nanotubes and Winkler elastic foundation modulus on the fundamental natural frequencies for the Euler- Bernoulli beam is investigated and tabulated. The governing differential equations are solved by using differential transform method. The differential transform method is applicable for nanotubes and gives high accuracy results.
\end{abstract}

Keywords: Aerospace, Winkler, DTM, DWCNT, MATLAB, MEMS.

○ RASĀYAN. All rights reserved

\section{INTRODUCTION}

Nanoscience and nanotechnology have made a principal contribution to the introduction of small-scale structures and devices. Iijima's discovery paper on multi-walled carbon nanotubes in $1991{ }^{1}$ pulled the attention of scientific researcher's communities in physics, chemistry and engineering. Some of the potential applications of nanorods and nanobeams are image technology, microelectro-mechanical systems (MEMS) and nanoelectromechanical systems (NEMS).,3

In Aerospace industries, There is a much need for new materials which exhibit improved mechanical properties i.e, high strength to weight ratio, high thermal stability and high corrosion resistance. Carbon nanotubes (CNTs) exhibit novel properties of having tensile strength in Tera pascal range, thermally stable up to $2800^{\circ} \mathrm{C}$ and vibration withstanding capabilities in the range of Tera Hertz. The structure of a single walled carbon nanotube can be visualized by packaging a single-atom-thick layer of graphite called graphene into a flawless cylinder. ${ }^{2-5}$

By now, there are three methods to analyze the mechanical properties of CNT. They are spectroscopy experiment, computational simulation and theoretical analysis. However, technically these are difficult experiments of vibrational properties because of the small diameter of the nanotubes, and plenty of time will be spent on the molecular dynamics (MD) simulation. Theoretical analyses using elastic continuum models have shown the advancement on the taken cost and the spent time of researches compared with experiment operation and MD simulation methods, and they can predict some results which cannot be achieved by the other two methods as of now. ${ }^{6-10}$

In the present work, DTM has been used to analyze the vibration of carbon nanotubes embedded on a Winkler foundation. Zhou ${ }^{11}$ developed differential transformation method for solving both linear and nonlinear initial value problems in electric circuit analysis. Later on Chen and $\mathrm{Ho}^{12}$ used this method to Eigen value problems.

\section{Differential Transform Method}

\section{EXPERIMENTAL}

The differential transform method is a semi-analytical method based on the Taylor series expansion. In this technique, certain transformation rules are used in converting the governing differential equations and the boundary conditions of the system in to a set of algebraic equations in terms of the differential

Rasayan J. Chem., 10(2), 481 - 487(2017)

http://dx.doi.org/10.7324/RJC.2017.1021711 
transforms of the original functions. The solution of these algebraic equations provides the results with a high degree of precision.

The differential transformation of the $\mathrm{i}_{\text {th }}$ derivative of the function $\mathrm{u}(\mathrm{x})$ is defined as follows:

$U(\mathrm{i})=\frac{1}{\mathrm{i} !}\left[\frac{d^{i} u(x)}{d x^{i}}\right]_{x=x_{0}}$

And the differential inverse transformation of $U(k)$ is expressed as:

$u(x)=\sum_{i=0}^{\infty} U(\mathrm{i})\left(x-x_{0}\right)^{i}$

In real application function, $\mathrm{u}(\mathrm{x})$ is expressed as finite series and equation (2) can be written as:

$u(x)=\sum_{i=0}^{n} U(k)\left(x-x_{0}\right)^{i}$

We can use the following transformation table for converting a differential equation in to the DTM form.

Table-1: Differential Transformations for Mathematical Equations

\begin{tabular}{c|c}
\hline Original Function & Transformed Function \\
\hline$y(x)=u(x) \pm v(x)$ & $Y(x)=U(\mathrm{i}) \pm V(\mathrm{i})$ \\
\hline$y(x)=\lambda u(x)$ & $Y(\mathrm{i})=\lambda U(\mathrm{i})$ \\
\hline$y(x)=\frac{d^{n} u(x)}{d x^{n}}$ & $Y(\mathrm{i})=(\mathrm{i}+1)(\mathrm{i}+2) \ldots .(\mathrm{i}+n) U(\mathrm{i}+n)$ \\
\hline
\end{tabular}

The superiority of the DTM is its simplicity and good precision and it takes less time to solve polynomial series ${ }^{11}$. It is different from the traditional high order Taylor's series technique, which needs symbolic computation of the necessary derivatives of the data functions. The Taylor series method takes relatively long time for computations involving large orders. With DTM technique, it is also possible to compute near exact solutions for differential equations.

\section{Mathematical Formulation}

Using the Euler-Bernoulli beam model, the required governing partial differential equation for motion of a beam is given by: ${ }^{8}$

$\rho A \frac{\partial^{2} w}{\partial t^{2}}+E I \frac{\partial^{4} w}{\partial x^{4}}=P(\mathrm{x})$

Where, $\mathrm{x}$ and $\mathrm{t}$ are the spatial coordinates. $\mathrm{w}$ is the deflection of carbon nanotubes and $\mathrm{P}$ is the transverse force acted on the nanotubes. E and I are the Young's modulus of elasticity and the mass moment of inertia, respectively. A is the area of cross section and $\rho$ is the density of nanotubes.

By using the Equation (4) governing equations for inner and outer nanotube can be written as:

$$
\begin{aligned}
& \rho A_{1} \frac{\partial^{2} w_{1}}{\partial t^{2}}+E I_{1} \frac{\partial^{4} w_{1}}{\partial x^{4}}=p_{1} \\
& \rho A_{2} \frac{\partial^{2} w_{2}}{\partial t^{2}}+E I_{2} \frac{\partial^{4} w_{2}}{\partial x^{4}}=p_{2}
\end{aligned}
$$

Where, the subscripts 1 and 2 denote the properties of the inner and outer nanotubes respectively. $p_{j}(\mathrm{j}=1,2)$ are the pressures exerted on inner and outer nanotubes. The pressure $\mathrm{P}_{1}$ acting on the inner 
nanotube due to thevdW interaction is given by,

$p_{1}=c\left(w_{2}-w_{1}\right)$

Where, $\mathrm{c}$ is the Vander Waals interaction coefficient between inner and outer nanotubes.

The pressure acting on the surface of outer nanotube due to presence of the Winkler medium can be given by,

$$
p_{w}=-k w_{2}
$$

Where, negative sign represents that $p_{w}$ is acting in opposite direction to the deflection of nanotubes. $k$ is the spring's stiffness.

Thus, for the DWCNTs, the pressure of the outer nanotube contacting with the Winkler medium is given by,

$$
p_{2}=p_{w}-c\left(w_{2}-w_{1}\right)
$$

In the model Van der Waals interaction (vdW) coefficient (c) can be obtained from the interlayer energy potential, given as He XQ et al ${ }^{14}$,

$$
c=\frac{\pi \varepsilon R_{1} R_{2} \sigma^{6}}{a^{4}}\left[\frac{1001 \sigma^{6}}{3} H^{13}-\frac{1120}{9} H^{7}\right]
$$

Where,

$$
H^{m}=\left(R_{1}+R_{2}\right)^{-m} \int_{0}^{\pi / 2} \frac{d \theta}{\left(1-K \operatorname{Cos}^{2} \theta\right)^{m / 2}},(m=7,13)
$$

and $K=\frac{4 R_{1} R_{2}}{\left(R_{1}+R_{2}\right)^{2}}$

Here $\mathrm{a}=0.142 \mathrm{~nm}$ (carbon to carbon atom bond length). $\mathrm{R}_{1}, \mathrm{R}_{2}$ are the inner and outer radii of double walled carbon nanotubes. $\sigma=0.34 \mathrm{~nm}, \varepsilon=2.967 \mathrm{meV}$ are the vdW radius and the well depth of LennardJones potential respectively as given by Saito et al. ${ }^{18}$

Thus,

$$
\begin{aligned}
& \rho A_{1} \frac{\partial^{2} w_{1}}{\partial t^{2}}+E I_{1} \frac{\partial^{4} w_{1}}{\partial x^{4}}=c\left(w_{2}-w_{1}\right) \\
& \rho A_{2} \frac{\partial^{2} w_{2}}{\partial t^{2}}+E I_{2} \frac{\partial^{4} w_{2}}{\partial x^{4}}=-k w_{2}-c\left(w_{2}-w_{1}\right)
\end{aligned}
$$

In this simulation, we consider the deflection of double walled carbon nanotubes has different vibration modes, $W_{j}(x), \mathrm{j}=1,2$ for the inside and outside nanotubes respectively. The displacements of the vibration analysis in double walled carbon nanotubes can be given by

$$
w_{j}(x, t)=W_{j}(x) e^{i \omega t}
$$

So equations (11) and (12) can be further simplified as:

$$
\begin{aligned}
& \frac{d^{4} \bar{W}_{1}}{d X^{4}}-\Omega^{2} \bar{W}_{1}=\beta\left(\bar{W}_{2}-\bar{W}_{1}\right) \\
& \delta \frac{d^{4} \bar{W}_{2}}{d X^{4}}-\eta \Omega^{2} \bar{W}_{2}=\beta\left(\bar{W}_{1}-\bar{W}_{2}\right)-\bar{k} \bar{W}_{2}
\end{aligned}
$$


Where,

$\Omega^{2}=\frac{\rho A_{1} \omega^{2} L^{4}}{E I_{1}}, \beta=\frac{c L^{4}}{E I_{1}}, \eta=\frac{A_{2}}{A_{1}}, \delta=\frac{I_{2}}{I_{1}}, \bar{k}=\frac{k L^{4}}{E I_{1}}, X=\frac{x}{L}$

\section{Application of DTM}

In converting the governing equations in DTM formulation, we use Table-1 and we can conveniently write the following expressions:

$$
\begin{aligned}
& W_{1}(\mathrm{i}+4)=\frac{\left(\beta \cdot\left(W_{2}(\mathrm{i})-W_{1}(i)\right)+r \cdot W_{1}(\mathrm{i})\right)}{(\mathrm{i}+1)(\mathrm{i}+2)(\mathrm{i}+3)(\mathrm{i}+4)} \\
& W_{2}(\mathrm{i}+4)=\frac{\left((\beta / \delta) \cdot\left(W_{1}(\mathrm{i})-W_{2}(\mathrm{i})\right)+r(\eta / \delta) \cdot W_{1}(\mathrm{i})-(\bar{k} / \delta) \cdot W_{2}(\mathrm{i})\right)}{(\mathrm{i}+1)(\mathrm{i}+2)(\mathrm{i}+3)(\mathrm{i}+4)}
\end{aligned}
$$

Where,

$$
r=\frac{\rho A_{1} \omega^{2} L^{4}}{E I_{1}},\left[\mathrm{k}=0.1,2,3 \ldots . . \mathrm{N} \_ \text {max }\right]
$$

Using the appropriate boundary conditions and transformed boundary conditions for DTM, the recurrence Eq. (16) and (17) can be solved for vibration frequencies for various modes.

\section{Boundary Conditions}

\section{Simply Supported CNTs}

For the simply supported CNTs beam boundary conditions at both ends are defined mathematically as:

$$
\mathcal{W}_{1}=0, \frac{d^{2} \mathcal{W}_{1}}{d x^{2}}=0, \mathcal{W}_{2}=0, \frac{d^{2} \mathcal{W}_{2}}{d x^{2}}=0
$$

\section{Clamped- Clamped CNTs}

For clamped-clamped CNTs, the boundary conditions at both ends are defined as:

$$
w_{1}=0, \frac{d}{d x}=0, w_{2}=0, \frac{d}{d x}=0
$$

\section{Clamped-Hinged CNTs}

For clamped-hinged CNTs case, the boundary conditions are defined as:

at $\mathrm{x}=0$

$$
w_{1}=0, \frac{d}{d x}=0, w_{2}=0, \frac{d}{d x}=0
$$

at $\mathrm{x}=\mathrm{L}$

$$
\mathcal{W}_{1}=0, \frac{d^{2} \mathcal{W}_{1}}{d x^{2}}=0, \boldsymbol{W}_{2}=0, \frac{d^{2} \mathcal{W}_{2}}{d x^{2}}=0
$$

\section{Comparison with Analytical Solutions}

\section{RESULTS AND DISCUSSION}

In this study, we consider double walled carbon nanotubes mounted in a Winkler medium having diameter of the inner and outer nanotubes of $4.8 \mathrm{~nm}$ and $5.5 \mathrm{~nm}$, respectively. The thickness of each nanotube is taken to be $0.34 \mathrm{~nm}$. The carbon nanotube has a Young's modulus of $1 \mathrm{TPa}$ and the density of $2.3 \mathrm{gm} / \mathrm{cm}^{3,21}$ 
By using the DTM as the numerical method the natural frequency for DWCNTs has been computed. Results are then compared with T.Natsukiet $a l^{21}$ and Xuet al. ${ }^{15}$ Very good agreement is observed with the T.Natsuki et al and Xu et al results for the simpy supported DWCNTs. We have taken $n=50$ so that the result converges up to four decimal places, where $n$ is the number of iterations required to converge the result.

Table-2: Simply supported (S-S) DWCNTs Fundamental frequency in THz

\begin{tabular}{c|c|c|c|c|c|c}
\hline Mode & $\omega_{1}$ & $\omega_{2}$ & $\omega_{3}$ & $\omega_{4}$ & $\omega_{5}$ & $\omega_{6}$ \\
\hline Present [DTM] & 0.618 & 2.586 & 5.447 & 7.283 & 7.564 & 8.248 \\
\hline T.Natsukiet al. ${ }^{21}$ & 0.62 & 2.60 & 5.50 & 7.40 & 7.70 & 8.50 \\
\hline Xu et al. ${ }^{15}$ & 0.635 & 2.652 & 5.450 & 7.30. & 7.65 & 8.45 \\
\hline
\end{tabular}

From the above Table-2, it is clearly observed that initial in-phase modes are matching perfectly and higher modes are slightly differing. As we observe that there is very little increment in vibration frequency after mode 4 , as vibration deflection will be in opposite direction of both nanotubes.

\section{New Results for DWCNTs}

Table-3 is the new extended results for the Clamped-Hinged (C-H) and Clamped- Clamped (C-C) boundary conditions.

Table 3: Fundamental frequency for the DWCNTs in THz

\begin{tabular}{l|l|l|l|l|l|l}
\hline Mode & $\omega_{1}$ & $\omega_{2}$ & $\omega_{3}$ & $\omega_{4}$ & $\omega_{5}$ & $\omega_{6}$ \\
\hline $\mathrm{C}-\mathrm{C}$ & 0.968 & 2.840 & 5.601 & 7.684 & 7.892 & 8.695 \\
\hline $\mathrm{C}-\mathrm{H}$ & 1.080 & 3.048 & 5.988 & 8.245 & 8.526 & 9.424 \\
\hline
\end{tabular}

It is observed that double walled carbon naotubesshow very high frequency over Terahertz. Modes 1 to 4 represent the first four vibration modes, which are in-phase modes, which means deflection of the inner and outer nanotubes are in the same direction. Modes 5 to 6 are the two vibrations of anti-phase mode, in which the deflections of the inner and outer nanotubes are in the opposite directions.

\section{Influence of variation of Length of DWCNTs on vibration frequencies}

Now if we change the length of nanotubes, vibration frequencies vary as:

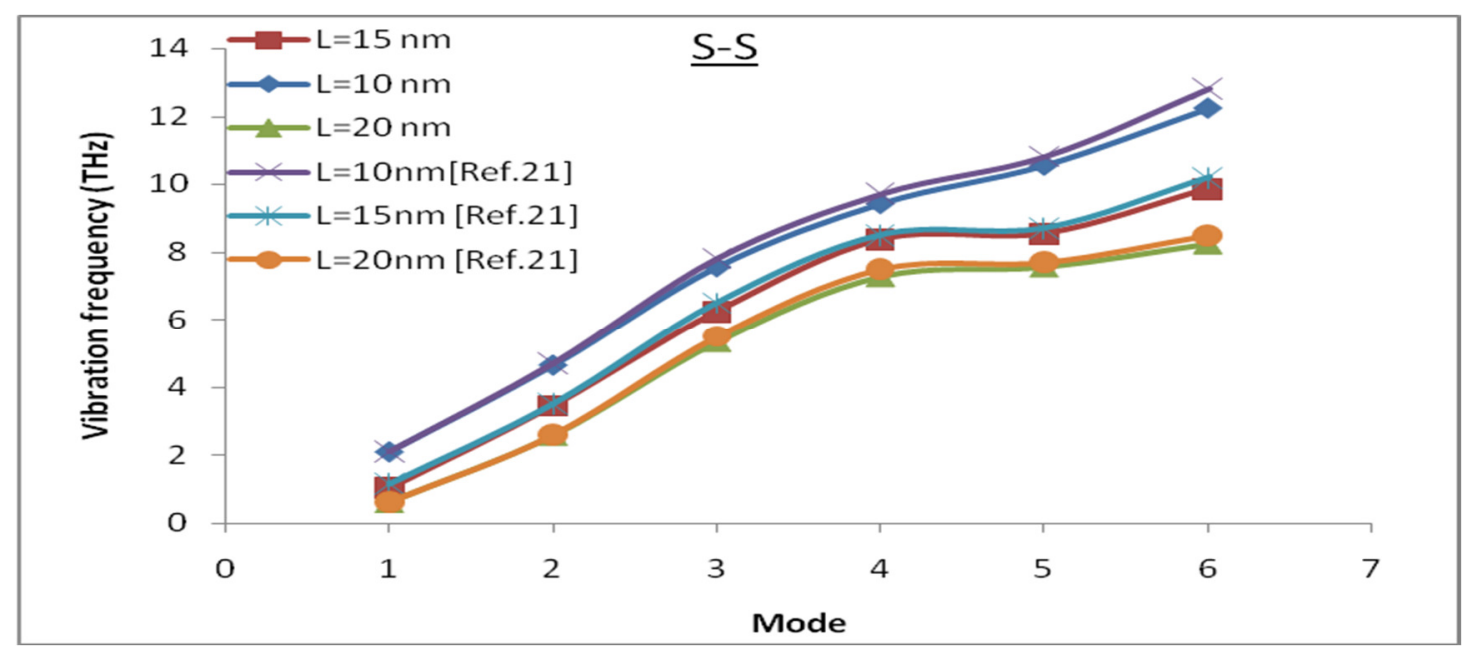

Fig.-1: Variation of vibration frequencies with length of DWCNTs for Simple supported boundary condition. Figures- 1, 2 and 3 show the variation of the vibrational frequncy with the length of nanotube for the various modes. Vibration frequnecy with the first mode in simply-supported DWCNTs is about 2.096 
$\mathrm{THz}$ from DTM which is very close to the available reference result ${ }^{21}$ with length of $10 \mathrm{~nm}$. It is clearly observed that with the increase of length of CNTs vibrational frequencies decrease. This effect is occuring due to the strong influence of phase modes on vibration frequencies.

\section{CONCLUSION}

In this study, the vibration investigation of DWCNTs embedded in an elastic medium for various boundary conditions like clamped-clamped, simply supported, and clamped hinged has been presented by using a semi-analytical numerical technique called the differential transform method in a simple and accurate way. The solution of the present vibration analysis problem using the DTM involves transformation of the governing equations of motion into algebraic set of equations and solving the transformed equations. Results show that phase modes dominate on vibration frequencies of CNTs. The analysis presented could be helpful in the application of CNTs in high-frequency oscillators, dynamic mechanical analysis and micro mechanical sensors.

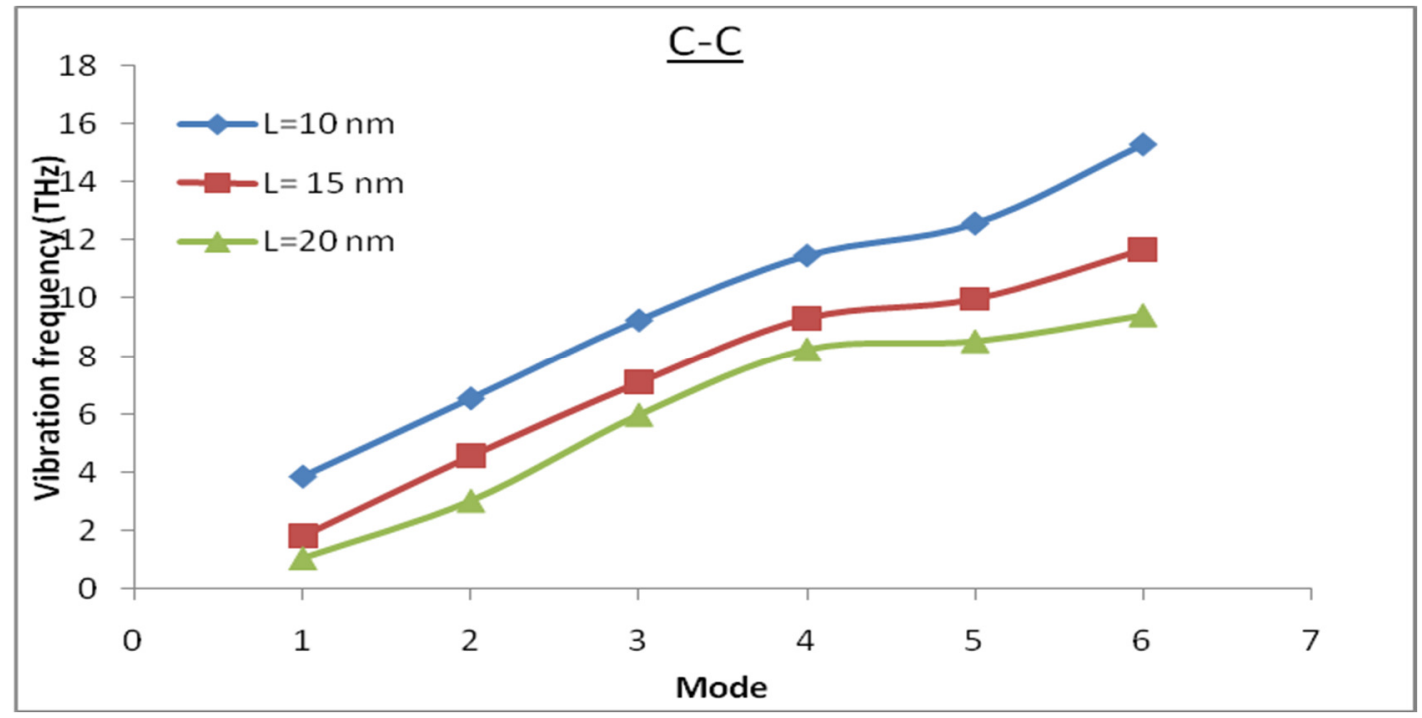

Fig.-2: Variation of vibration frequencies with Length of Clamped-Clamped DWCNTs

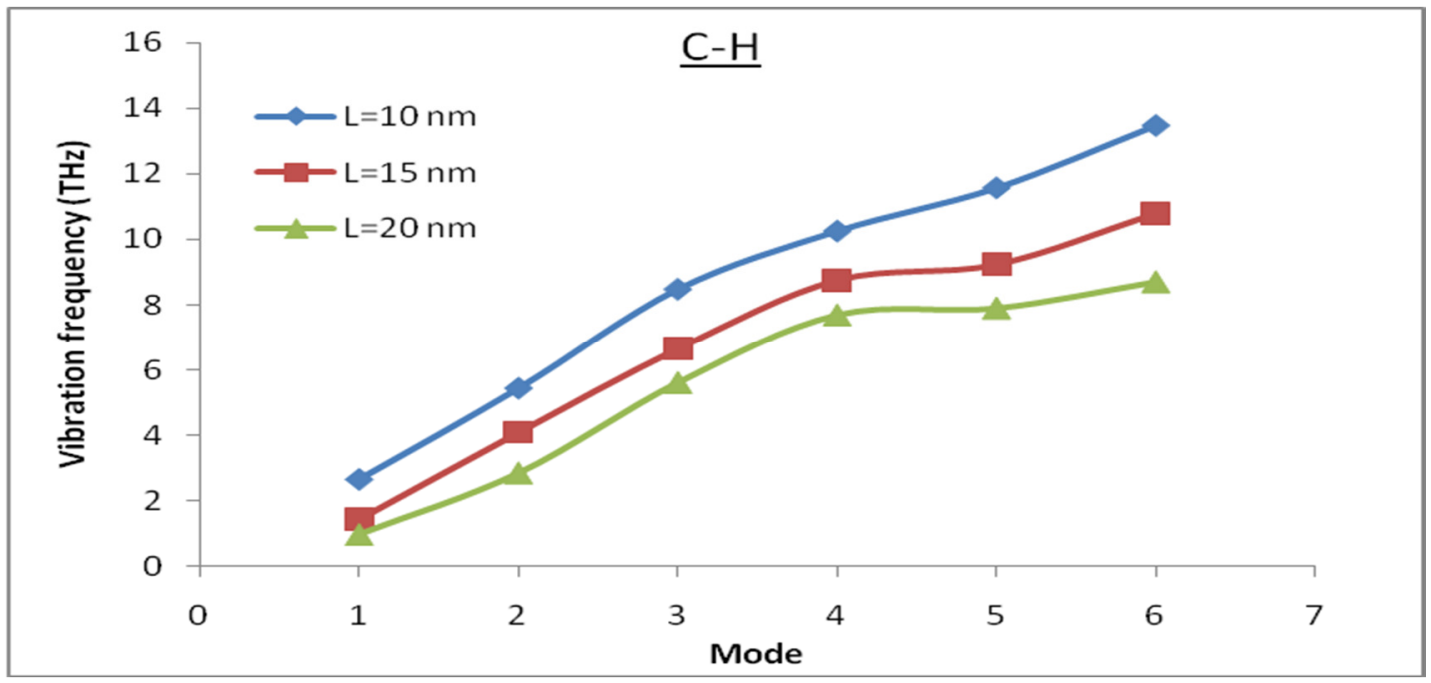

Fig.-3:Variation of vibration frequencies with Length of Clamped-Hinged DWCNTs 


\section{REFERENCES}

1. S. Iijima, Nature, $\mathbf{5 8}, 354$ (1991).

2. P. Ball, Nature, 414, 6860, (2001).

3. X. Wang, Q. Li, J. Xie, Z. Jin, J. Wang, Y. Li and S. Fan, Nano letters, 9(9), 3137(2009).

4. E. T. Thostenson, Z. Ren and T. W. Chou, Composites science and technology, 61(13), 1899(2001).

5. E. Pop, D. A. Mann, K. E. Goodson and H. Dai, Journal of Applied Physics, 101(9), 093710 (2007).

6. M. J. Treacy, T. W. Ebbesen and J. M. Gibson, Nature, 381(6584), 678(1996).

7. Q. Wang and V. K. Varadan, Smart Materials and Structures, 14(1), 281(2005).

8. I. Elishakoff and D. Pentaras, Journal of Sound and Vibration, 322(4), 652(2009).

9. R. Kumar and S. Deol, Nonlocal Buckling Analysis of Single-Walled Carbon Nanotube Using Differential Transform Method (DTM). International. J. Sci. Res., 5, (2016).

10. J. Yoon, C. Q. Ru, A. Mioduchowski, ASME Journal of AppliedMechanics,72, 10(2005).

11. J. K. Zhou, Huazhong Science \& Technology University Press, China (1986).

12. C. O. K. Chen and S. H. Ho, Applied Mathematics and computation, 106(2), 171(1999).

13. B. Ravi Kumar, Intenational Journal of ChemTech Research, 10(3), 339(2017).

14. XQ. He, S. Kitipornchai, CM. Wang, KM. Liew, Int J Solids Struct,42(23), 6032(2005).

15. K. Y. Xu, E. C. Aifantis, Y. H. Yan, ASME Journal of Applied Mechanics,75(2),021013(2008).

16. V. M. Harik, Solid State Commun., 120, 331(2001).I

17. C. Li, and T. W. Chou, Appl. Phys. Lett.,84, 121(2004).

18. R. Saito, R. Matsuo, T. Kimura, G. Dresselhaus, M. S. Dresselhaus, Chern.,Phys.Lett., 348, 187 (2001).

19. Shakti Singh, G. K. Lohiya, and V. K. Mourya, Rasayan J. Chem., 2(2), 451(2009).

20. V. S. Angulakshmi, S. Karthikeyan et. al., Rasayan J. Chem., 8(1), 1(2015).I

21. T. Natsuki, Q. Q Ni, M. Endo, CARBON, 46, 1570(2008).

[RJC-1711/2017] 